\title{
Signal Coverage Estimation Model for Microcellular Network Propagation
}

\author{
${ }^{1}$ J. J. Biebuma, ${ }^{2}$ B.O.Omijeh, ${ }^{3} \mathrm{M}$.M.Nathaniel \\ ${ }^{1,2,3}$ Dept. of Electronic and Computer Engineering, University of Port Harcourt, Port Harcourt, Rivers State. \\ E-mail: bourdillon.omijeh@uniport.edu.ng
}

\begin{abstract}
This paper presents the signal coverage estimation for microcellular network propagation. Firstly, this work presents the techniques for eliminating or reducing the drawbacks like limited service capability, high blocking probabilities and inefficient frequency spectrum utilization that negatively affect the signal network coverage in land to mobile networks and macro cellular networks due to wider or larger cell radii. Secondly, the methodology of improving signal coverage under different morphological conditions is also presented, and this was achieved by creating a communication path between two remote base stations under different morphological conditions. The signal strength received in all the different morphologies are then estimated for different cell radii. Thirdly, the results of the estimated signal strength in all the morphologies are also presented in this paper. The results show that the signal strength received in urban morphology is the strongest because, the distance between the cell radii of the base stations are closer than sub-urban and rural morphologies. Lastly, this research also reveals the importance of using signal strength measurements, path loss and cell radius to determine signal coverage estimation for micro cellular network propagation. Finally, it shows that the reduction of the cell radius and path loss will automatically increase the received signal strength, which helps in achieving the accuracy of signal coverage prediction of microcellular network under different morphologies.
\end{abstract}

Keywords: Morphology, microcell, macrocell Path loss, Cell radius,

\section{Introduction}

The concept of cellular telephony is largely attributable to developments in radiotelephony at the Bell Telephone Laboratories from late 1940s to the early 1970s, and these developments led to the first commercial cellular telephone services being launched in the early 1980s. Since that time, the growth and popularity of mobile telecommunications has been extraordinary. The capacity of cellular systems can be increased by splitting and sectoring existing cells, thereby reusing frequencies more often in a geographic area. In practice, however, there is a capacity limit as cells cannot be split indefinitely. The lower cell radius limit for most conventional cellular systems (herein referred to as "macro cells") is in the range of 1 to $4.5 \mathrm{~km}$. Microcell technologies are being developed to provide wireless communications to very large numbers of people at a much higher user density than is possible with macro cells. Microcell architecture differs from macro cell architecture in three fundamental ways: The cells are typically less than $1 \mathrm{~km}$ in radius. The mobile terminals radiate at much lower power levels[Bernadine et al, 1998]. All radio channels are available in every cell. The wide scale deployment of an extensive, high grade wireless telephone system will require engineering tools and techniques that allow rapid and accurate system design. The fundamental problem that needs to be addressed is modelling the end result of many users transmitting in a congested area. As the number of deployed microcells increases, site-by-site engineering may become too time consuming and costly. Yet service quality targets, including cell coverage and call blocking and dropping probabilities need to be predicted and met[Hata, 1980]. More so, signal coverage is an important factor to be considered while designing a cellular network for a particular morphology. A cellular network must be designed in such a way that the interferer will have a minimal effect on the transmission and reception of the signal. A called subscriber must have a clear assess to the calling subscriber, also a clear communication path is required between the two users of a cellular network. With the introduction of high-speed multimedia services, the demand for signal coverage has become more important. A prerequisite for improving signal coverage in a cost-efficient way is the detailed knowledge of a planned microcellular network coverage. This information can be obtained from predictions or measurements and estimation[Jeruchim et al, 1982] [Montgonery et al,1992]

\section{Related Works}

In their paper [Manoj, et al, 1998], presented that cellular systems are designed for certain cell edge or cell area reliability. To achieve this a shadow fade margin is included in the link budget to determine the effective cell radius[Okumura, et al, 1968]. But due to the finite number of measurements used to model the propagation, the mean signal strength at the estimated cell edge may be different from the actual desired mean 
signal strength [Reudink, 1974]. To design a system for required coverage reliability in the noise limited case, the design must include the variation of the signal due to lognormal shadowing and the effect of finite signal strength measurements[Giancristofaro,1996] [Coulson et al. 1998].

[Jeruchim, et al, 1982] in their work presented a new interference model for microcellular networks which integrates radio propagation parameters and user terminal mobility. Their model uses a parameter denoted the "interference to noise ratio" (INR) to obtain a simplified description of mobile link outage contours as a function of the location of the fixed and mobile radio ports[Sorensen. 1998]. The INR is used to demonstrate that microcell networks are more interference limited than macrocell networks, and thus are more affected by user terminal mobility [Gudmundson. ,1991].

The modern cellular systems are being built to provide high quality of service and high capacity. The typical cell radius in urban areas is less than one kilometer. To minimize the interference (co-channel and adjacent channel interference) it is important to determine the coverage boundary accurately [Sorensen, 1998]. Estimating inaccurate coverage has severe impact on the network performance.

Hata, 1980] in their paper indicated that the average received signal power decreases logarithmically with distance for outdoor radio channels.

The average large-scale path loss for an arbitrary transmit - receive (T-R) separation can be expressed as a function of distance[Okumura et al, 1968].

$$
\operatorname{PL}(\mathrm{d})=\mathrm{A}+\mathrm{Blog} \mathrm{do}
$$

PL denotes the average path loss at a distance $d$ from the transmitter and do is the close-in reference distance which is determined from measurements close to the transmitter ie the distance between the two base stations. When plotted on a log-log scale, the modeled path loss is a straight line with an intercept A with a slope equal to $\mathrm{B} \mathrm{dB}$ per decade. The values of $\mathrm{A}$ and $\mathrm{B}$ depend on several factors including the propagation environment, frequency of operation and transmitter height. In macro cellular systems where the cell coverage is large, reference distances of $1 \mathrm{Km}$ are commonly used. The reference distance should be in the far field of the antenna so that near field effects do not alter the reference path loss[Hata, 1980].

\section{Research Methodology}

The research methodology employed in this work involves three stages as follow:

1) Field survey and data collection in urban, sub urban and rural morphologies

2) respectively by using VHF Omni Directional Radio (VOR)

3) Data analysis by using Monte Carlos Simulation technique

4) Comparative analysis of signal Coverage characteristics in different morphologies

\subsection{Field Survey and Data Collection}

In carrying out this research, three location were visited for field survey and data collection. These areas are Port Harcourt Local Government Area (Urban Morphology), Eleme Local Government Area (Suburban Morphology) and Adoni Local Government Area (Rural Morphology). The collection of data were done in three stages as explained below.

\section{Step 1 Field survey and data collection in Urban morphology}

A Vhf Omni Directional Radio(VOR) that determines the positions and cell radius of a Base Station was place at a strategic position of less than $1 \mathrm{~km}$ between the two Base Stations in the axis of Port Harcourt L.G.A within the coverage of MTN Network. At a bin size of 35 meters, the VOR was switched on and the cell radius was determined as $0.4 \mathrm{~km}$. This measurement was repeated for bin sizes of $40 \mathrm{~m}, 45 \mathrm{~m} 50$ and $60 \mathrm{~m}$ respectively, and the corresponding cell radii were determined and recorded for all the bin sizes. These data are shown in table 1.

Table 1: Data collected from urban morphology

\begin{tabular}{|l|l|l|}
\hline No. of Bins & Bin size in meters $(\mathrm{m})$ & Cell radius $(\mathrm{Km})$ \\
\hline 1 & 35 & 0.40 \\
\hline 2 & 40 & 0.45 \\
\hline 3 & 45 & 0.50 \\
\hline 4 & 50 & 0.55 \\
\hline 5 & 60 & 0.60 \\
\hline
\end{tabular}




\section{Step 2 Field survey and data collection Suburban morphology}

A Vhf Omni Directional Radio(VOR) that determines the positions and cell radius of a Base Station was place at a strategic position of $2 \mathrm{~km}$ to $4.5 \mathrm{~km}$ between the two Base Stations in the axis of Eleme L.G.A, also within the coverage of MTN Network. At a bin sizes of 160m, 180m, $200220 \mathrm{~m}$ and 240m, the VOR was energized and switched on, and the cell radii were determined for the corresponding bin sizes. These data are shown in table 2 .

Table 2: Data collected from suburban morphology

\begin{tabular}{|l|c|c|}
\hline No. of Bins & Bin size in meters $(\mathrm{m})$ & Cell radius $(\mathrm{Km})$ \\
\hline 1 & 160 & 3 \\
\hline 2 & 180 & 3.4 \\
\hline 3 & 200 & 3.8 \\
\hline 4 & 220 & 4.0 \\
\hline 5 & 240 & 4.2 \\
\hline
\end{tabular}

Step 3 Field survey and data collection Rural morphology.

Also, A Vhf Omni Directional Radio(VOR) was also place at a strategic position of distance beyond $4.5 \mathrm{~km}$ between the two Base Stations in Adoni L.G.A within the coverage of MTN Network. At a bin size 500 meters, the VOR was switched on and the cell radius was determined and read from VOR as $9 \mathrm{~km}$. This measurement was repeated for bin sizes of $500 \mathrm{~m}, 600 \mathrm{~m}, 700 \mathrm{~m}, 800 \mathrm{~m}$ and $900 \mathrm{~m}$ respectively. The corresponding cell radii were determined for all the bin sizes and their values were recorded. These data are shown in table 3 .

Table 3: Data collected from rural morphology

\begin{tabular}{|l|l|l|}
\hline No. of Bins & Bin size in meters $(\mathrm{m})$ & Cell radius $(\mathrm{Km})$ \\
\hline 1 & 500 & 9.0 \\
\hline 2 & 600 & 10.0 \\
\hline 3 & 700 & 11.0 \\
\hline 4 & 800 & 12.0 \\
\hline 5 & 900 & 13.0 \\
\hline
\end{tabular}

From the three tables above, it is clear that the smaller the bin size the smaller the cell radius. Also, the wider the bin size the larger the cell radius. The relationship between the cell radius and the bin size is that the cell radius increases with the increase in bin size and vise visa. This show that cell radius is directly proportional to bin size

\subsection{Data Analysis using Monte Carlos Simulation technique}

Monte Carlos Simulation technique is a unique technique for studying the effect of the design reliability of cell radius and received signal under different environmental conditions i.e morphology, and to determine the minimum required strength of the received signal and cell radius that will be required for effective signal coverage of microcellular network. To achieve this, the data collected from the field were fed into Monte Carlos modules, and the simulation set up is discussed below.

To simulate the signal coverage conditions in urban, suburban and rural morphologies(i.e a cell that exists in reality), the following simulation setup were used as stated below:

(1). The entire cell area was divided into 9 different clutter blocks of several bins.

(2) The number of bins were chosen so that the measurement of cell radii with the corresponding bin sizes were taken for urban, suburban and rural cells.

Based on the experimental results of several investigators on correlation models [Gudmundson, et al, 1991], a grid of 12 × 12 bins was chosen. This would correspond to a bin size of 35 to 60 meters in an urban cell, 160 to 240 meters in a suburban cell and 500 to 900 meters in a rural cell. More so, each block of $12 \times 12$ bins is modeled to represent a different clutter such as a block of high rise buildings, mountains etc. Thus there are total of 9 different clutter blocks. The base station is assumed to be at the center of the square grid to determine the effects of environmental conditions on the received signal strength in each morphology. From Empirical Propagation Model, the average large scale path loss PL in decibels $(\mathrm{dB})$ for a distance d between the base stations i.e cell can be expressed as

$$
\mathrm{PL}=\mathrm{A}+\mathrm{B} \log (\mathrm{d})
$$


The parameters A and B are the intercept and slope of propagation model based on Hata's empirical results[Hata, 1980]. The assumed base station antenna height is 50meters, and a mobile station height is 1.5 meters and operating frequency of $800 \mathrm{MHz}$. Under these assumptions, the value of B is $35.22 \mathrm{~dB} / \mathrm{decade}$ and the value of $\mathrm{A}$ is $130 \mathrm{~dB}$ for urban morphology, $120 \mathrm{~dB}$ for suburban morphology and $110 \mathrm{~dB}$ for rural morphology. Under these conditions, the path loss PL at a distance $\mathrm{d}$ was determined by using equation 1 , The base station transmit power Pt was chosen to be $50 \mathrm{~W}$ which is typical transmitting power for cellular systems with Omni antenna configuration, and $\mathrm{d}$ is the cell radius. For this study, Omni antennas were considered though the results are applicable for sectored antennas also. The effect of antenna pattern is normalized and the received signal strength at any bins is calculated using

$$
\operatorname{Pr}=\mathrm{Pt}-
$$

\section{Determination of path loss in urban, suburban and rural morphologies}

Path loss(or Path attenuation) is the reduction in power density of an electromagnetic wave as it propagates through space. Path loss may due to many effects such as terrain contours, environment (urban or rural vegetation), distance between the transmitter and the receiver, and the height and location of antennas. Path loss for the three morphologies were determined as follows:

$\mathbf{P L}=(\mathbf{A}+\mathbf{B}) \cdot \log (\mathbf{d})$

$\mathrm{PL}=$ Path loss between the base stations

A = Intercept of propagation model based on Hata's empirical results

$\mathrm{B}=$ Slope of propagation model based on Hata's empirical model results(it has a constant value of 35.22dB/decade)

$\operatorname{Pr}=$ Received signal strength of the bins i.e received power in watt(W).

$\mathrm{Pt}=$ Transmitted power of the base station antenna in watt $(\mathrm{W})$, and is $50 \mathrm{~W}$ for base station

$\mathrm{d}=$ Cell radius kilometers $(\mathrm{km})$

$$
\begin{aligned}
& \mathbf{P L}=(\mathbf{A}+\mathbf{B}) \cdot \log (\mathbf{d}) \text { for Urban morphology } \\
& \text { Bin1, } \quad \mathrm{PL}=(130+35.22) \log 0.4=-65.8 \mathrm{~dB} \\
& \text { Bin2, } \quad P L=(130+35.22) \log 0.45=-57.3 \mathrm{~dB} \\
& \text { Bin3, } \quad P L=(130+35.22) \log 0.45=-49.7 d B \\
& \text { Bin4, } \quad \text { PL }=(130+35.22) \log 0.5=-42.9 \mathrm{~dB} \\
& \text { Bin5, } \quad P L=(130+35.22) \log 0.6=-36.7 \mathrm{~dB}
\end{aligned}
$$

From the results above, the path loss increase with increase in cell radius and it increases with increase in cell radius. The path loss also decreases with decrease in cell radius. This show that the cell radius is directly proportional to path loss.

\section{$\mathbf{P L}=(\mathbf{A}+\mathrm{B}) \cdot \log (\mathrm{d})$ for suburban morphology}

Bin $1, \quad$ PL1 $=(120+35.22) \log 3=74.5 \mathrm{~dB}$

Bin2, $\quad$ PL $=(120+35.22) \log 3.4=82.5 \mathrm{~dB}$

Bin3, $\quad$ PL $=(120+35.22) \log 3.8=89.9 \mathrm{~dB}$

Bin4, PL $=(120+35.22) \log 4.0=93.5 \mathrm{~dB}$

Bin5, $\quad$ PL $=(120+35.22) \log 4.2=96.7 \mathrm{~dB}$

From the results above, the path loss increase with increase in cell radius and it increases with increase in cell radius. The path loss also decreases with decrease in cell radius. This show that the cell radius is directly proportional to path loss.

$$
\begin{aligned}
& \mathbf{P L}=(\mathbf{A}+\mathbf{B}) \cdot \log (\mathbf{d}) \text { for rural morphology } \\
& \text { Bin } 1, \quad P L=(110+35.22) \log 9.0=138.6 \mathrm{~dB} \\
& \text { Bin2, PL }=(110+35.22) \log 10.0=145.2 \mathrm{~dB} \\
& \text { Bin3, PL }=(110+35.22) \log 11.0=151.2 \mathrm{~dB} \\
& \text { Bin4, PL }=(110+35.22) \log 12.0=156.7 \mathrm{~dB} \\
& \text { Bin5,PL= }(110+35.22) \log 13.0=161.8 \mathrm{~dB}
\end{aligned}
$$

From the results above, the path loss increase with increase in cell radius and it increases with increase in cell radius. The path loss also decreases with decrease in cell radius. This show that the cell radius is directly proportional to path loss.

\section{Determination of received signal strength in urban, suburban and rural morphologies.}

;Pr $=$ Pt-PL, where Pt $=$ Transmission power of the base station and was chosen to be $50 \mathrm{~W}$. It is assumed to be constant for all the morphology 


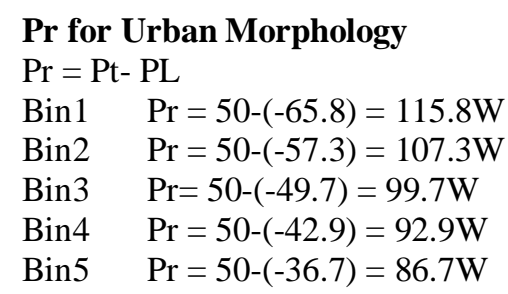

Pr for Suburban Morphology

$\operatorname{Pr}=\mathrm{Pt}-\mathrm{PL}$

Bin $1 \quad \operatorname{Pr}=50-74.5=-24.5 \mathrm{~W}$

Bin2 Pr $=50-82.5=-32.5 \mathrm{~W}$

Bin3 Pr $=50-89.9=-39.9 \mathrm{~W}$

Bin4 Pr $=50-93.5=-43.5 \mathrm{~W}$

Bin5 $\operatorname{Pr}=50-96.7=-46.7 \mathrm{~W}$

\section{Pr for Rural Morphology}

Pr $=$ Pt- PL

Bin $1 \quad \operatorname{Pr}=50-138.6=-88.6 \mathrm{~W}$

Bin2 $\operatorname{Pr}=50-145.2=-95.2 \mathrm{~W}$

Bin3 Pr $=50-151.2=-101.2 \mathrm{~W}$

Bin4 Pr $=50-156.7=-106.7 \mathrm{~W}$

Bin5 Pr $=50-161.8=-111.8 \mathrm{~W}$

\subsection{Comparative analysis of signal Coverage characteristics in different Morphologies}

The comparative analysis of the signal coverage characteristics of urban, suburban and rural morphologies was achieved as stated below.

(1) Comparative analysis of signal coverage in urban, suburban and rural morphologies with respect to the effect of cell radius and path loss.

(2) Comparative analysis of signal coverage in urban, suburban and rural morphology with respect to effect of path loss and received signal strength.

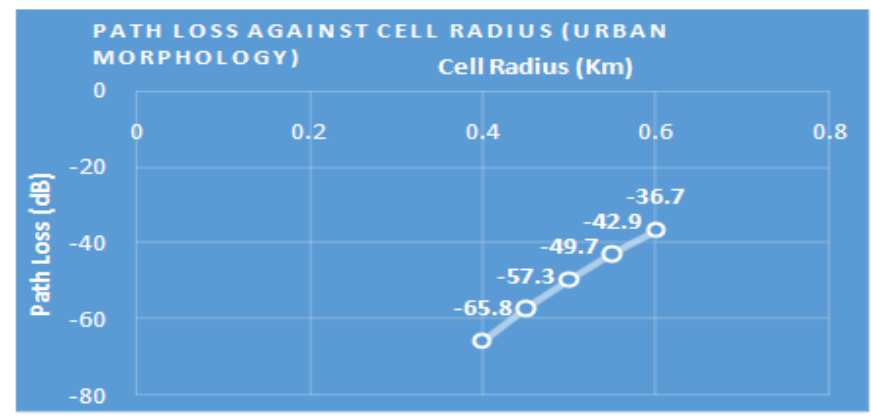

Fig.1: The effects of cell radius and path loss on signal coverage in urban morphology

From Fig.1, it noticed that cell radius and path loss have common relationship. The cell radius increase with increase in path loss, and also the cell radius decrease with decrease in pathloss

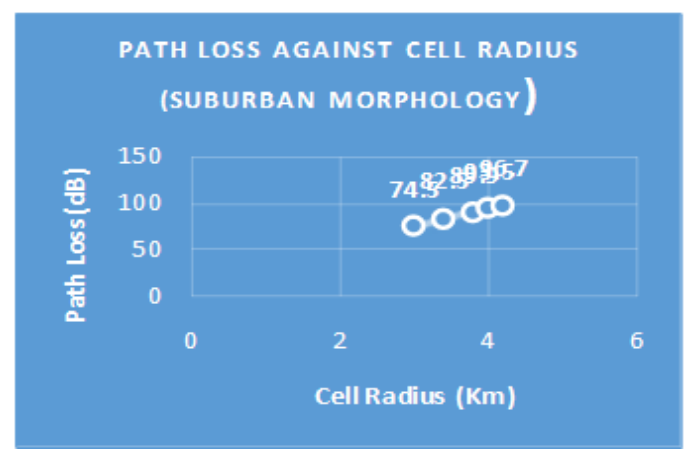

Fig.2: The effects of cell radius and path loss on signal coverage in suburban morphology

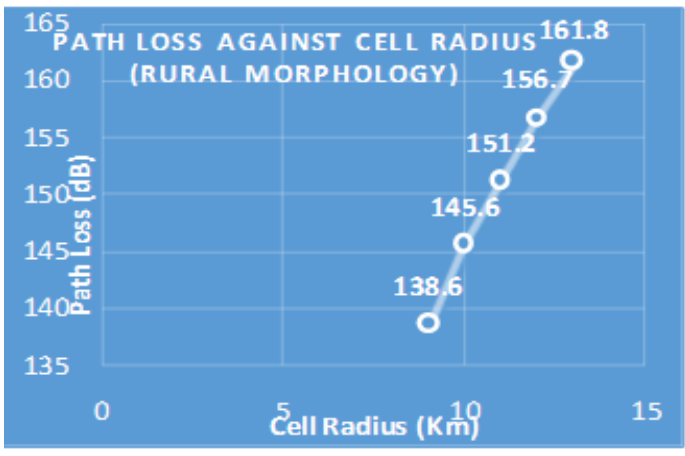

Fig. 3: The effects of cell radius and path loss on signal coverage in rural morphology 
The curve in Fig 2, also shows that the cell radius increase with increase in path loss.

The curve in Fig.3 shows that the cell radius is directly related to path loss. When the cell radius is $9 \mathrm{~km}$ the path loss is $138.6 \mathrm{~dB}$, and when the cell radius increase to $13 \mathrm{~km}$ the path loss also increase to $161.8 \mathrm{~dB}$.

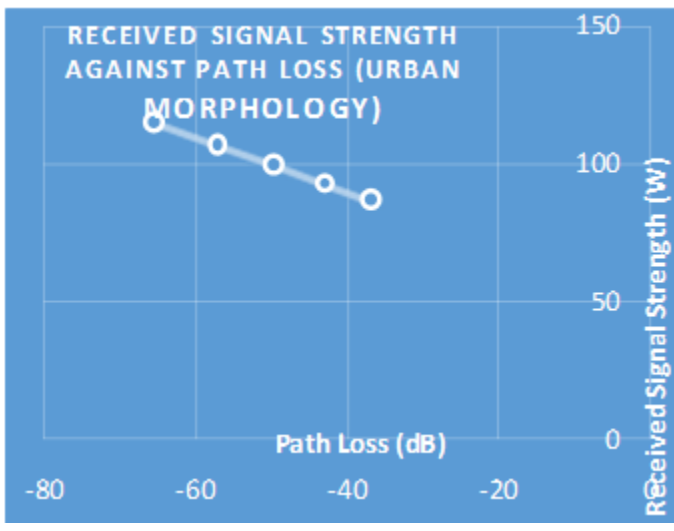

Fig.4: The effects of received signal strength and path loss on signal coverage in urban morphology

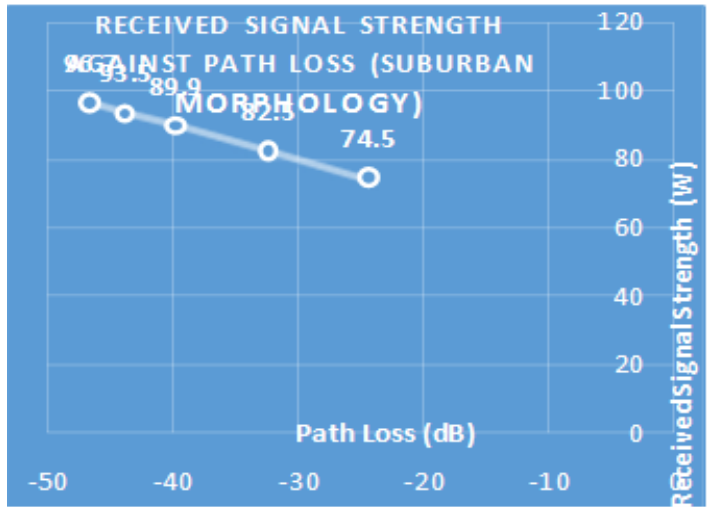

Fig.5 The effects of received signal strength and path loss on signal coverage in suburban morphology

The curve in Fig.4 shows that the path loss increases with decrease in received signal strength, and also decreases with increase in received signal strength.

From Fig.5, the curve, the received signal strength increases from $-46.7 \mathrm{~W}$ to- $24.5 \mathrm{~W}$, while the path loss decreases from $96.7 \mathrm{~dB}$ to $74.5 \mathrm{~dB}$.

The graph in Fig.6 shows that large values of path loss will reduce the strength of the signal in the network. The behavior of the curve shows that the cell radii are at the widest range of all the three morphologies, hence, the largest values of path loss were recorded. The effect of path loss on the signal coverage will reduce the received signal strength to the smallest values.

In summary, the signal strength received in urban morphology is the strongest, hence a very good signal coverage is achieved in urban morphology than suburban and rural morphologies respectively.

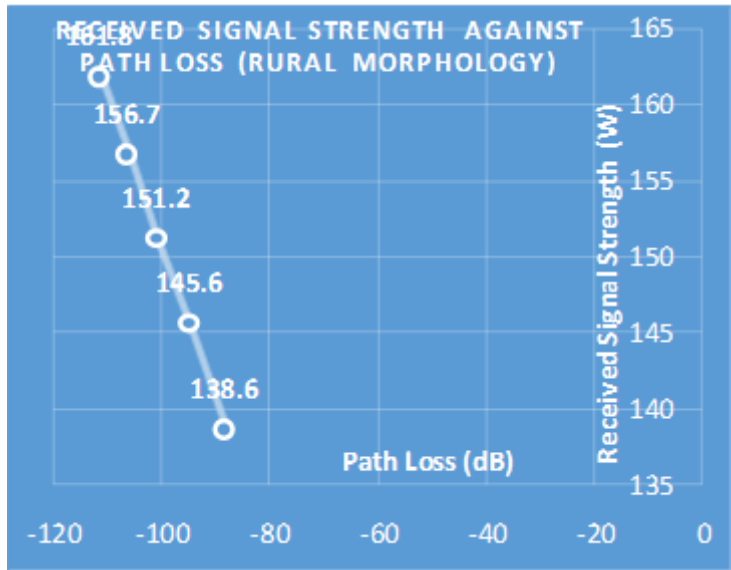

Fig.6: The effects of received signal strength and path loss on signal coverage in rural morphology

\section{Results And Discussion}

The results that were obtained from the research are stated and explained below:

(1). The effects of the power received, Path loss and cell radius on signal coverage in urban morphology. The effects of received signal strength, path loss and cell radii on signal coverage in urban morphology are shown and explained in Fig.7. 


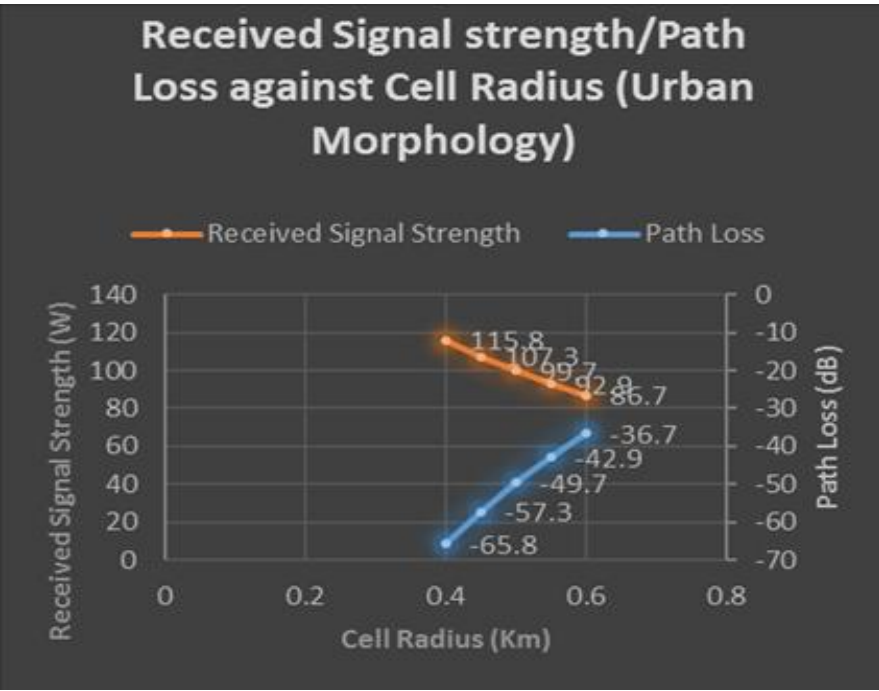

Fig.7: The effects of the power received in base station, Path loss and cell radii on signal coverage in urban morphology

From Fig.7, the cell radii are increasing with increase in path loss, while the cell radii reduce as the received signal strength increase. By reading the graph above, when the received signal strength is increasing from $86.7 \mathrm{~W}$ to $115.8 \mathrm{~W}$, the cell radii will decrease from $0.6 \mathrm{~km}$ to $0.4 \mathrm{~km}$. Also, when the path loss increase from $-65.8 \mathrm{~dB}$ to $-36.7 \mathrm{~dB}$, the cell radii also increase from $0.4 \mathrm{~km}$ to $0.6 \mathrm{~km}$. This relationship show that increment in cell radii and path loss will reduce the signal strength received for effective coverage signal in micro cellular network, while the reduction in cell radii and path loss increases the received signal strength in micro cell base stations

(2) The effects of the power received Path loss and cell radii on signal coverage in suburban morphology. The effect of power received, path loss and cell radii on signal coverage of cellular network are explained in fig 8 below

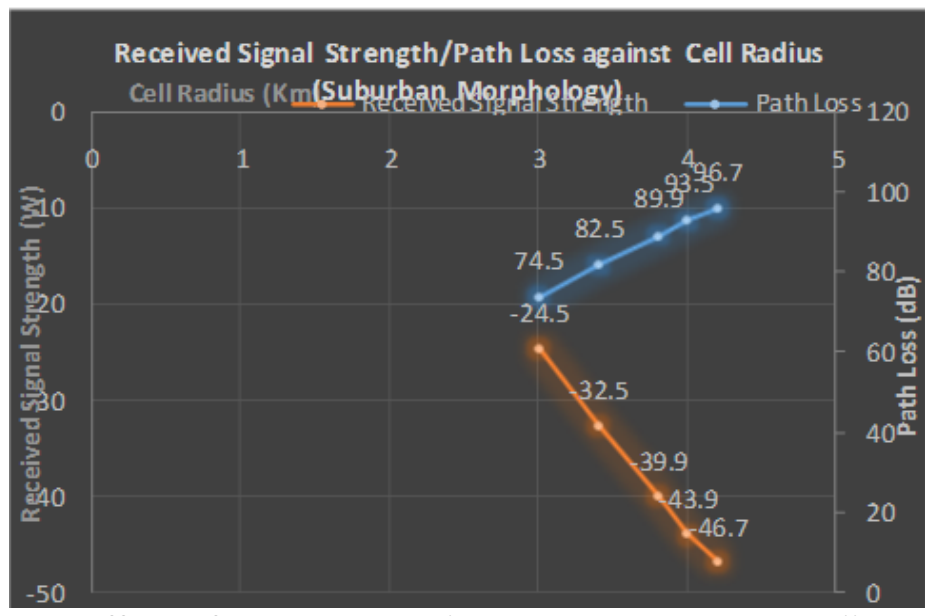

Fig .8: The effects of the power received, Path loss and cell radii on signal coverage in suburban morphology

The graph above shows that the cell radii increase with reduction in received signal strength, hence the path loss become lager that the values obtained in fig 4.1 . As the path loss increase from $74.5 \mathrm{~dB}$ to $96.7 \mathrm{~dB}$, the received signal strength continue to decrease from $-24.5 \mathrm{~W}$ to $-46.7 \mathrm{~W}$. The consequence of this is that the receive signal strength become weaker as the cell radii and path loss increase, and this will limit the fidelity of the signal received.

(3) The effects of the power received, Path loss and cell radii on signal coverage in rural morphology. The effect of cell radii and path loss on received signal strength in rural morphology is the main factor that determines the signal coverage in rural morphology. This factor is explained in fig.9, 


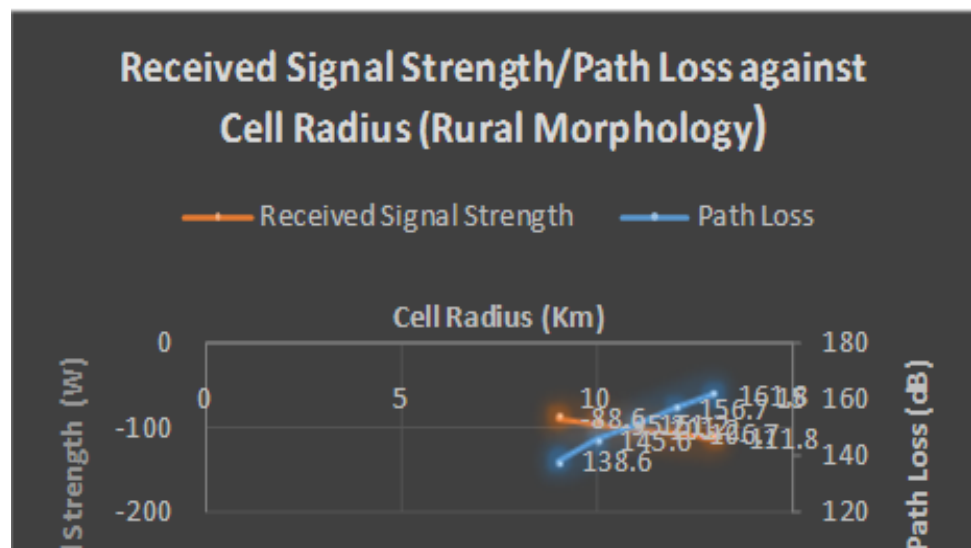

Fig.9: The effects of the power received, Path loss and cell radii on signal coverage in rural morphology

From the graph in fig.9, the cell radii increase with largest values from $9 \mathrm{~km}$ to $13 \mathrm{~km}$, and this cause path loss to increase at the same rate. Consequently, the received signal strength is reduced drastically from $88.6 \mathrm{~W}$ to $-111.8 \mathrm{~W}$ which are not suitable for effective signal coverage in micro cellular network propagation. Also from the graph, when the received signal strength is lower than $92.5 \mathrm{~W}$, no signal will be received in the network coverage area ,because the path loss is so appreciable to make the signal fade off.

In summary, from all the three characteristics of the curves for urban suburban and rural morphologies respectively, fig.7, shows that before an effective signal coverage can be achieved in micro cellular network, the cell radius must be relatively small so that the path loss can be reduced to the values that will allow effective received signal strength in the micro cellular network.

(4) The comparative effects of received signal strength, path loss and cell radius on signal coverage in urban, suburban and rural morphologies.

From fig 7,8 and 9 above, it has been established that cell radius increases with path loss at the expense of received signal strength, while the received signal strength increases at the expense of cell radius and path loss.. It can be seen that ell radii increase with increase in path loss at the expense of received signal strength. In urban morphology, the received signal strength increase from $86.7 \mathrm{~W}$ to $115.8 \mathrm{~W}$ up the curve, while the path loss and cell radii decrease from $-36.7 \mathrm{~dB}$ to $-65.7 \mathrm{~dB}$ and $0.6 \mathrm{~km}$ to $0.4 \mathrm{~km}$ down the curve respectively. Also, from suburban morphology, the received signal strength increase from $-46.7 \mathrm{~W}$ to- $24.5 \mathrm{~W}$ up the curve at the expense of path loss and cell radii that decrease from $96.7 \mathrm{~dB}$ to $74.5 \mathrm{~dB}$ and $3 \mathrm{~km}$ to $4.2 \mathrm{~km}$ respectively. The curve also shows that the received signal strength increase from $-111.8 \mathrm{~W}$ to

$-88.6 \mathrm{~W}$ up the curve with both path loss and cell radii decrease from $138.6 \mathrm{~dB}$ to $161.8 \mathrm{~dB}$ and $9.0 \mathrm{~km}$ to $13.0 \mathrm{~km}$ respectively down the slope in rural morphology. From the curve of urban morphology, when the base stations are $0.40 \mathrm{~km}$ apart, the received signal strength is $115.8 \mathrm{~W}$. Also from the curve of suburban morphology, when the base station are $3 \mathrm{~km}$ apart the signal received becomes $-24.5 \mathrm{~W}$. This is also applicable to rural morphology, when the base stations are $9.0 \mathrm{~km}$ apart the received signal strength becomes $-88.6 \mathrm{~W}$. The implication of this effect is that base station cell radii decrease with increase in received signal strength. Also the received signal in urban morphology is the strongest while the received signal strength in rural morphology is the weakest. This results can be traced to the disparity in their cell radii.

Hence, the signal coverage in urban morphology becomes most reliable and dependable of all the three morphologies considered in this research.

\section{Conclusion}

This thesis has shown the importance of using signal, path loss and cell radius measurement to determine signal coverage estimation for micro cellular network propagation. The relations between cell radius cell, signal strength, and path loss are very significance to effective signal coverage in microcellular network. From the methodology of this research, the effect of reducing the cell radius will automatically increase the received signal strength, and this helps in achieving the accuracy of signal coverage prediction of microcellular network under different morphologies. From the results, effective signal coverage can be achieved by disintegrating the morphology under a network coverage into smaller cells of a very small radius of less than $0.4 \mathrm{~km}$ apart. This will help the telecommunication engineer to minimize the effect of signal attenuation that affects the received signal strength in cellular network. The received signal strength must be large enough to accommodate the effects of path loss 


\section{Reference}

[1]. Bernardin. M, Yee. M. and Ellis. T, (1998) "Cell Radius Inaccuracy: A New Measure of

[2]. Coverage Reliability," IEEE Transactions on Vehicular Technology, vol 47, No 4 , pp. 1215-1226

[3]. Coulson, A. J, Williamson. A. G. Vaughan R. G. (1998) “A statistical basis for lognormal

[4]. Shadowing effects in multipath fang channels", IEEE Transactions on Communications,

[5]. vol. 46, no 4

[6]. Giancristofaro. D. (1996) "Correlation model for shadow fading in mobile radio channels", IEE

[7]. Electronics Letters, vol. 32, no. 11, pp. 958-959.

[8]. Gudmundson. M. (1991) "Correlation model for shadow fading in mobile radio systems", IEE Electronics Letters, vol. 27, pp. 2145-2146

[9]. Hata. M. (1980) "Empirical Formula for Propagation Loss in Land Mobile Radio Services,"

[10]. IEEE Transactions on Vehicular Technology, vol VT 29.

[11]. Hata .M, Kinoshita. K, Hirade. K (1982) "Radio link Design of Cellular Land Mobile Communication system”, IEEE Transactions on Vehicular Technology, vol VT 31

[12]. Jeruchim. M.C. Balaban. P. and Shanmugan. K. Lee. W.C.Y, (1982) Mobile Communications Engineering, McGraw Hill Book Co., New York, , P. 104

[13]. Manoj. K, Bernardin. P and Tamil. L. (1998) "Coverage predication for cellular networks from limited signal strength measurements", Processing of IEEE International symposium on personal, indoor and mobile radio communication, pp. 1147 1151 .

[14]. Montgonery. D.C, and Peck. E.A (1992) "Introduction to Linear Regression Analysis, Services". Edition John Willey \& Sons, Inc.

[15]. Okumura. Y. et al., (1968) "field strength and its variability in UHF and VHF land mobile radio Service," Rev. eLec. Commun. Lab. Vol 16, pp 825-873.

[16]. Reudink. D.O (1974) "properties of mobile radio prorogation above 400MHz," IEEE Transaction on Vehicular technology, vol VT23,Pp 143-160.

[17]. Sorensen. T. B, (1998) "Correlation model for slow fading in a small urban macro cell", Proceedings of IEEE International Symposium on Personal, Indoor and Mobile Radio Communications, pp. 1161-1165, (September.

\section{Authors' Biography}

Biebuma Joel Jeremiah holds a B.Eng \& M.Sc (S.C.T, Sussex, England) in Electronic \& Communication Engineering, \& Ph.D (S.C.U, California, U.S.A) in Telecommunication Engineering . He is a member, Nigeria Association for Teachers of Technology (MNATT), Member Institute of Electronics and Electrical Engineers (MIEEE) and Member, Nigeria Society of Engineers. His research interest is in Electronics, Telecommunication, Antennas, Microwaves etc. He is a prolific writer with numerous technical papers and engineering text books. He is currently a Senior Lecturer and Ag. HOD, Department of Electronic and Computer Engineering, University of Port Harcourt, Port Harcourt, Nigeria. He is happily married with Children

Bourdillon .O. Omijeh holds a B.Eng degree in Electrical/Electronic Engineering, M.Eng and Ph.D in

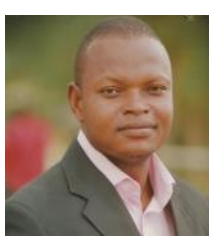
Electronics/Telecommunications Engineering from the University of Port Harcourt \& Ambrose Alli University (A.A.U) Ekpoma, respectively. His research areas include: Artificial Intelligence, Robotics, Embedded Systems Design, Modeling and Simulation of Dynamic systems, Intelligent Metering Systems, Automated Controls, Telecommunications and ICT. He has over thirty (30) technical papers \& publications in reputable National \& International peer reviewed Journals. He has authored some Electrical/Electronic Engineering Text books; and also, has developed over ten(10) engineering application Software. He is a member, Institute of Electronics and Electrical Engineers (MIEEE), Corporate Member, Nigeria Society of Engineers; and also, a registered practicing Engineer with COREN. He is currently a Senior Lecturer \& pioneer HOD, Department of Electronic and Computer Engineering, University of Port Harcourt, Nigeria; and also, a consultant to companies \& Institutions. He is happily married with Children.

Micah.M.Nathaniel holds a B.Eng. Degree of Electrical Engineering from University of Ilorin, Nigeria. He has

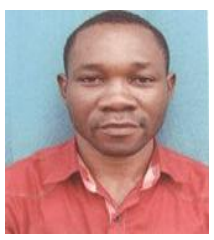
a wide experience in Telecommunication Engineering, Technical sales and service delivery.. $\mathrm{He}$ is a master student in the department of Electrical/Electronic Engineering, University of Port Harcourt. His research interest are : Telecommunication, Electronic, ICT and Control. 